\title{
Notes sur la crise de la régulation étatique
}

\section{Xavier ARBÓs}

Universitat de Barcelona

Working Paper n.39

Barcelona 1991 
A partir du moment où l'on décide de définir comme champ de recherche celui de la régulation, on est obligé d'élargir la perspective habituelle de chacune de nos spécialités. On est obligé d'admettre que l'analyse du droit positif n'est pas suffisante à ce propos, car on accepte que la régulation se fait aussi par des voies différentes de celles de la législation ou la réglementation. Mais il me semble que le fait même que la régulation soit devenue un sujet problématique peut mieux se comprendre en raison de l'incapacité de l'État contemporain à établir son autorité dans la société. L'empire de la loi, le rule of law, n'est plus effectif. L'ordre social n'est plus le résultat de la règle de droit, si jamais l'a-il été. Mais tout de même, elle y est encore décisive. Si la règle de droit étatique montre sa faiblesse (parce qu'on a voulu la pousser trop loin, peut-être), il est aussi vrai qu'elle garde encore une certaine force pour rendre acceptables les "autres" formes de régulation, celles qui composent le droit non étatique. Cette force reste tout de même importante, car c'est la règle de droit étatique qui peut faire mobiliser les ressources de la puissance publique pour rendre efficace le droit non étatique. Cependant, il faut rappeler que la réflexion sur ces problèmes n'est pas du tout récente. Durkheim avait déjà signalé en 1897 l'incapacité de l'État de gérer efficacement la vie professionnelle, et il proposait une régulation non étatique. Et, en 1909, Santi Romano décrivait aussi l'impuissance du droit public: "Il diritto pubblico moderno dunque non domina, ma è dominato da un movimento sociale, al quale si viene stentatamente adattando, e che intanto si governa con delle leggi proprie". La crise de l'État moderne serait due à la convergence de deux facteurs: l'organisation des intérêts dans la société et le manque d'instruments juridiques et institutionnels adaptés (1).

Les sociétés occidentales présentent des tendances qui s'orientent vers une mise en cause du rôle de l'État et de sa fonction régulatrice. Depuis que son pouvoir a été investi de la légitimité démocratique, en le laissant prendre soin de l'intérêt général, cette notion même commence à s'évanouir comme bien d'autres fictions (2). Le réalisme nous fait reconnaître l'importance des groupes d'intérêt sous toutes leurs formes, et l'on se demande s'il ne serait pas convenable de leur laisser le soin de réguler des domaines déterminés (3), au nom de l'expertise et de l'efficacité. C'est un choix dont je traiterai à la fin. Préalablement, je me propose de rappeler les éléments qui définissent la crise de l'État contemporain, et de décrire les effets de cette crise sur la régulation. Plus précisément, je prendrai comme modèle de référence l'État providence, et dans une deuxième partie j'essaierai de présenter des liens entre la crise de l'État et la recherche de nouvelles formes de régulation.

\section{LA CRISE DE L'ÉTAT PROVIDENCE}

II n'est pas question d'analyser tous les aspects de la crise, mais d'en 
souligner les dimensions qui touchent de plus près la fonction normative de l'État. C'est dans ce sens qu'il est intéressant de rappeler un diagnostic du cadre général de la crise des démocraties, paru en 1975 (4). "La crise des démocraties" y est perçue comme le résultat de la surcharge (5) du système politique, qui est incapable de répondre à toutes les demandes que lui sont formulées. Selon une analyse assez habituelle, la liberté politique permet l'expression des demandes, et la participation démocratique leur donne des voies d'accès aux institutions et de l'influence sur les décideurs. Mais le système risque le blocage ou la fermeture, car les recours ne suffisent jamais à satisfaire toutes les demandes, qui, en plus, sont assez souvent contradictoires. Et ce qu'on demande aux pouvoirs publics comporte presque toujours l'intervention dans le marché en réduisant sa liberté. La tentation, alors, est celle de limiter toute restriction au marché et même les demandes qui pourraient y conduire (6).

Toute cela encadre la spécificité de l'État providence. Les problèmes sont ceux qui viennent d'être énoncés, mais aggravés dans la mesure où les pouvoirs publics ont le devoir (légal ou même constitutionnel) de mettre en oeuvre des politiques publiques destinées à satisfaire au moins des besoins jugés essentiels, tels que la santé ou l'éducation. Bien entendu, cette spécificité n'arrive pas à donner une originalité absolue au modèle contemporain d'État providence (7). L'ensemble des politiques publiques qui ont leur trait distinctif peut être établi depuis Bismarck en Allemagne, et il a été présent dans beaucoup d'États sous des gouvernements différents. Ironiquement, on a pu dater son origine en 1883, l'année de la mort de Marx et de la naissance de Keynes (8). Sur un plan plus général, il semble clair aussi que le comportement réel des États libéraux ne s'est jamais limité au domaine qu'en principe on lui croyait propre, celui d'un "veilleur de nuit" (9).

Ceci dit, il y a tout de même des éléments qui permettent de définir l'État providence. Ainsi, on peut dire que ce sont les pouvoirs publics qui développent le rôle essentiel "protecteur" (10), et que ce rôle est parallèle à la notion de droits sociaux (11). L'État providence (12) est censé agir en ayant des finalités d'émancipation, de libération des individus des situations de nécessité qu'ils ne peuvent pas surmonter de leurs propres forces. Je ne suis pas sûr qu'en français on puisse faire la différence entre "liberté pour" et "liberté de"; il est assez commun en anglais de signaler comme un trait distinctif des conceptions associées à l'État providence sa lutte pour "the freedom from want" (13). L'action des pouvoirs publics, qui offrent des services et interviennent sur le marché, obéit à des prescriptions légales. Et ce modèle avance dans sa consolidation au moment où les droits sociaux sont constitutionnalisés: c'est I'"État social" (14) que l'on trouve (parfois explicitement) dans les constitutions européennes nées après la deuxième guerre mondiale (15).

Depuis la fin des années 70 , il y a un consensus assez répandu sur la crise 
de l'État providence. II apparaît comme un modèle dont la crise est irréversible, car elle est le résultat de certaines politiques sociales qui ont un effet négatif sur l'économie. Le diagnostic du directeur des affaires sociales de I'OCDE, était pessimiste en 1980, mais au moins on pouvait en tirer un message clair: du point de vue de l'économie, le modèle d'État providence ne marchait plus (16). L'interventionnisme des pouvoirs a acquis des connotations négatives et la tendance libérale à présenter l'État comme le Leviathan dangereux et oppressif par nature se répand. C'est ainsi qu'on peut lire dans la préface d'un livre sur l'État que le sujet central est celui d'expliquer "how state and society interact to disappoint and render each other miserable" (17).

Du point de vue du droit, l'État providence n'a pas été toujours bien accepté par tous les juristes dans ce qui constitue son originalité. Face au mouvement ouvrier des débuts du siècle, nous signale Weber, les juristes qui lui étaient favorables trouvaient des difficultés à traduire les demandes sociales dans le langage normatif du moment. Le formalisme du droit (du droit libéral) fut mis en question et il fallut passer souvent au champ éthique de la justice matérielle, opposé a celui de la légalité formelle (18). La constitutionnalisation de l'État providence dans la forme d"'État social" culmine le processus de transformation du droit libéral qui cesse de pouvoir être défini par sa neutralité. Les droits sociaux et la notion même d'État social sont devenus des catégories acceptables dans la vie du droit, leur étant reconnue la nature juridique. Mais il y a toujours des réticences qui signalent très raisonnablement les dangers d'une politisation de l'application du droit (19). Laissons de côté le doute sur la possibilité qu'il y ait un droit non susceptible d'être considéré politisé (20); les potentialités du droit de l'État social ne peuvent pas se réaliser du moment où la culture juridique continue à voir la Constitution comme la limite du pouvoir en négligeant ce qu'il y a de programme pour les pouvoirs publics (21).

\section{LA CRISE DE LA FONCTION RÉGULATRICE}

La crise de la fonction régulatrice apparaît dans le cadre d'une société où de larges domaines de la vie se trouvent "juridifiés" (22), souvent avec des réglementations particulières. Ceci ne comporte pas nécessairement la négation de l'existence d'espaces de "non-droit", au sens donné par J. Carbonnier (23), mais la constatation du fait qu'il y a des prévisions normatives qui, appliquées ou non, touchent des événements autrefois considérés strictement privés (comme la maladie), ou qui se trouvaient réglées par des dispositions contractuelles génériques du code civil. Cet élargissement du domaine de la régulation étatique produit une série de problèmes. Le premier est quantitatif: la prolifération normative accroît la complexité du système normatif. Deuxièmement, la forme régulatrice par excellence qui est la loi a aussi subi des transformations que j'aborderai après. Pour 
terminer cette partie, j'essaierai de décrire les problèmes d'efficacité qui s'opposent à la régulation étatique.

\section{a) Surproduction et complexité}

L'État providence souffre de "surproduction" législative (24). C'est la réponse à la "surcharge" de demandes sociales dont je parlai au début de ce travail. Une réponse obligée dans le cadre d'un État qui est toujours un État de droit, où l'action des pouvoirs publics est légitimée par des normes juridiques et le législateur est le représentant de la volonté populaire (25). La production normative de l'État providence est énorme, et les gouvernements conservateurs des années 80 ne l'ont pas réduite (26) de façon significative. Les pouvoirs publics doivent agir, mais ils doivent le faire dans le respect des normes, et cela devient compliqué quand celles-ci sont trop nombreuses. L'exigence du respect du principe de légalité peut se heurter à la capacité de réaction efficace des pouvoirs publics, ce qui est le cas là ou la Constitution n'établit pas un domaine réservé au règlement et n'admet pas des règlements praeter legem (27). Il est vrai que le constitutionnalisme contemporain ne limite pas le pouvoir normatif au parlement, et admet la délégation législative dans certains cas (28). Néanmoins, le problème se pose et il semble plus difficile aux États-Unis, où l'idée même d'un pouvoir normatif hors du Congrès est difficile à admettre (29), et il a fallu accepter les régulations des Independent Regulatory Commissions. II faut souligner que ces commissions ne se trouvent pas sous la hiérarchie du Président, et le Congrès a vu la Cour Suprême lui refuser la légitimité constitutionnelle de ses instruments de contrôle sur ces commissions (30). II serait intéressant de voir jusqu'à quel point tout le débat lancé aux États-Unis peut s'expliquer par le rejet des normes qui se produisent sans contrôle ni légitimation des élus. D'autre part, et dans un plan général, il y a un important élément d'inertie dans la législation. Une nouvelle majorité parlementaire ne change pas toujours la législation précédente, même si les forces politiques qui arrivent au pouvoir avaient voté contre lorsqu'elles étaient dans l'opposition (31).

Ajoutons à ce panorama deux particularités des États de notre entourage, où l'on voit augmenter la complexité du système juridique. La première, c'est le droit communautaire, dont les normes posent souvent des problèmes d'application. La deuxième, c'est la tendance à la diffusion de modèles politiques proches $d u$ fédéralisme. En principe, ces deux phénomènes ne devraient pas comporter des difficultés insurmontables pour les États. Ceux-ci acceptent de transférer la fonction régulatrice dans des cas précis à des instances supranationales ou régionales, mais cela produit des problèmes dès que les normes "supra" ou "infra" étatiques se heurtent à la forme régulatrice par excellence qui est la loi de l'État. Celle-ci a cessé d'avoir une seule source, car, en Espagne par exemple, il y a des lois étatiques produites par le législateur "national" et des lois aussi étatiques produites par le 
législateur "régional". La clarté d'un certain modèle libéral où la loi pouvait se présenter comme "expression de la volonté générale" (32)"est plus difficile à trouver. Certes, on peut dire que la diffusion du pouvoir normatif étatique l'a enrichie en légitimité. Mais ce gain de légitimité ne se trouve pas dans l'existence du droit communautaire, qui s'impose à toute forme de droit étatique (33).

\section{b) Transformation de la loi}

La surproduction crée la complexité de l'ordre normatif contemporain, et elle est aussi la dimension la plus visible de la crise de la fonction régulatrice. Mais cette crise a aussi un profil interne, plus proche du juriste, qui est celui du changement du modèle de loi. La loi n'est plus caractérisée par la portée générale de son mandat. La législation contemporaine présente un nombre élevé de "lois spéciales" et de "lois singulières", qui ont une portée délibérément limitée (34). Les lois spéciales se heurtent à la conception libérale du droit de façon très directe: elles rétrécissent la liberté contractuelle et elles s'orientent vers des buts qui s'imposent aux décisions des individus. Elles deviennent souvent des "status" pour les groupes sociaux auxquels la loi s'adresse et qui trouvent dans la loi spéciale l'instrument pour arriver à des buts qui, sans elle, auraient dû être obtenus par voie de contrat dans le cadre général du code civil. Elles sont aussi le résultat des négociations entre les groupes d'intérêt et les autorités publiques (35), et ce genre de loi, à la limite, conformerait un État de droit qui serait celui du "gouvernement des intérêts privés". La littérature sociologique en anglais a attribué a ce genre de gouvernement le sigle P.I.G. (Private Interest Government), d'une expressivité un peu trop poussée. En tout cas, ce genre de législation semble correspondre par son inégalité au modèle de société corporative qui domine les pays occidentaux (36). En ce qui concerne la loi singulière, elle peut avoir une portée encore plus réduite, voire individuelle. La loi singulière n'établit pas seulement une réglementation juridique précise (et non pas générale); elle peut arriver à désigner les cas concrets où elle doit s'appliquer (37).

Les lois spéciales et les lois singulières posent des problèmes communs, plus aigus dans le cas des lois singulières, en ce qui concerne l'idée même d'État de droit. Si l'on prend la position qui identifie l'État de droit à la garantie de la liberté individuelle, la perte du caractère général et abstrait de la loi est considérée comme une dénaturalisation du rôle même de la loi; l'autodétermination individuelle se développe par la voie du contrat, et cette voie est bloquée à partir du moment où la loi tient compte des cas particuliers. La loi gagne en arbitrariété ce qu'elle perd en abstraction (38). Le fait que les lois spéciales ou particulières puissent répondre à des mandats de la Constitution, surtout dans ce qu'elles peuvent avoir de mandat au législateur de diriger l'action des pouvoirs publics vers des objectifs d'égalité matérielle (39), ne fait que transférer ces objections de la loi à la Constitution. Si l'on tend à identifier l'État de droit à la démocratie, dans la mesure où la loi est le produit 
d'un Parlement représentatif (40), l'élaboration presque contractuelle de la loi avec les groupes d'intérêt se contredit avec la notion de loi comme expression de la volonté générale, ou même, si l'on veut, de la majorité des citoyens. D'autre part, l'attribution à la volonté générale ou à une majorité de citoyens devient une fiction de plus en plus difficile à accepter. Les négociations comportent la réduction de la transparence, de la loi et la perte de la publicité de sa production que la vision libérale de la loi considérait importante (41). Et, en plus, du point de vue de la technique législative (42), ces lois négociées avec les intéressés posent des problèmes importants. La procédure se prolonge par le besoin d'écouter des organisations, qui, à leur tour, doivent créer des accords internes. Cette prolongation de la procédure peut être le prix à payer pour obtenir un consensus qui serait la garantie de l'efficacité d'une loi ainsi élaborée, ayant été le fruit du consensus parmi les secteurs les plus concernés. Mais l'intérêt social global peut demander avant tout une réponse immédiate, et la qualité technique de la loi (sa cohérence interne et par rapport au système normatif) peut dégénérer en un patchwork dont on ne sait pas très bien assurer l'applicabilité. D'ailleurs, il n'est pas sûr que l'applicabilité des lois et leur efficacité soient toujours le souci principal des législateurs; ils sont soumis à la tentation de faire des "lois-spectacle" qui assurent la satisfaction de l'opinion publique mais qui accroissent, parfois innécessairement, la complexité du système normatif (43).

La transformation de la loi et la perte de sa généralité ont amené le droit à une situation de fragmentation où les "status" abondent (44). Georges Burdeau avait présenté ce problème dans un cadre où les Constitutions n'avaient pas de force effective à gérer la vie collective. On voit que le problème subsiste même dans les cas où l'efficacité de la Constitution est garantie par les Cours constitutionnelles (45). En plus, la dynamique de l'ordre normatif s'est accélérée sans logique unitaire de développement, dans l'espoir de répondre aux demandes que la société produit et que les groupes d'intérêt présentent. La stabilité n'est plus une caractéristique du droit, comme l'on pouvait croire aux temps où le code civil régnait presque en solitaire. II devient plus difficile de connaître le droit applicable et la sécurité juridique en souffre. La "certitude de la loi" (46) devra être recherchée au-dessus d'elle: "La certezza di ieri era fondata su ciò che la norma diceva; la certezza di oggi, su ciò che la norma ordinaria non può dire (per il controllo di legittimità costituzionale), o deve dire (in adempimento di obblighi, imposti dalle norme costituzionali agli organi legislativi)" (47). Dans ce sens, il faut souligner que la certitude du droit, ainsi que la rationalité et la cohérence du système juridique, ne semblent être possibles qu'après la formulation définitive de la norme par le biais du contrôle juridictionnel de la constitutionnalité. Au cas où ce contrôle n'a lieu qu'après l'entrée en vigueur de la loi, les décisions sur la constitutionnalité des lois ont normalement des effets ex nunc (48), même en République fédérale d'Allemagne. 
La fonction régulatrice étatique se trouve donc dans une crise qui peut être attribuée à la complexité de l'ordre normatif, accrue par le rôle des cours constitutionnelles. On pourrait présenter l'État contemporain comme étant de temps en temps paralysé par deux forces contradictoires: d'une part, celle des limites imposées par l'univers normatif qu'il a produit et que les pouvoirs publics sont tenus de respecter chaque fois qu'ils veulent mettre en oeuvre une décision (49); de l'autre, par les demandes qui lui sont présentées par des électeurs qui pourront juger les gouvernants tant sur le plan du respect des normes que sur celui de l'efficacité. La légalité est une composante de l'État de droit, où le pouvoir public est exercé dans le respect des normes; dans ce sens, elle est une composante essentielle de la légitimité. Mais la légitimité s'établit aussi par rapport à l'efficacité de l'action des pouvoirs publics (50), et du moment où cette action se fait à travers des normes, il faut aussi réfléchir au lien entre les normes et l'efficacité des décisions qu'elles expriment.

\section{c) Efficacité de la régulation étatique}

Le problème de l'efficacité est au centre des débats parmi les différents courants de la "science juridique" (51), et la possibilité de l'aborder me semble hors de la portée de ce travail. Mais on peut tout de même évoquer le problème du degré selon lequel les acteurs politiques sont déterminés par des normes. La question a été posée de façon particulièrement aiguë en relation à l'État totalitaire nazi, étant le contraire d'un État de droit, par le titre d'un article d'Otto Kirchheimer, qui qualifiait l'État de droit comme "mur magique" (52). Mais la considération du droit comme "rempart" met en premier plan la garantie des libertés. Le problème de l'efficacité du droit, du droit comme "outil" (53), est celui de l'État de droit qui essaie de réduire l'arbitraire dans l'ensemble d'interventions qu'il multiplie sur la société, comme réponse aux demandes que celle-ci lui adresse. On pourrait dire que l'action du pouvoir politique se développe sub specie iuris dans un contexte où la performance technique est un élément secondaire (54). Ce qu'on juge d'une décision juridifiée, c'est sa validité et la régularité de sa mise en oeuvre. Mais la rationalité juridique ne correspond pas à la rationalité technique. Pour prendre les modèles wéberiens, le droit agirait à partir de la rationalité formelle, tandis que l'efficacité serait dépendante de la rationalité matérielle ou technique (55). L'ordre normatif est hiérarchisé de façon rigide, tandis que les régulations techniques s'articulent selon les problèmes à résoudre sans former de système hiérarchisé. On pourrait dire que la caractéristique essentielle est l'utilité et non pas la validité.

Rien d'étonnant, donc, si le législateur délègue la concrétisation de ses mandats aux experts de l'Administration qui sont censés être capables de formuler la règle la plus adaptée à l'obtention d'un résultat pratique, et qui connaissent les cibles qui doivent être touchées. La circulaire est un instrument utilisé fréquemment, 
malgré les difficultés que pose son efficacité externe sur les particuliers (56). Quand les destinataires extérieurs sont les banques privées ou la Bourse, il est évident que l'importance des circulaires est énorme (57) . La régulation qui en résulte n'est plus une régulation étatique au sens traditionnel: elle ne s'insère pas facilement dans le contexte de la vision traditionnelle de la hiérarchie normative, qui ne prévoit pas les effets extérieurs des circulaires, et surtout quand I'habilitation bénéficie des autorités qui jouissent d'une grande indépendance. Les exemples espagnols qu'on peut présenter sont la Banque d'Espagne et la Commission Nationale du Marché des Valeurs mobilières. Dans ces cas, et dans d'autres comparables, les auteurs de la régulation ne sont pas soumis à l'autorité politique qu'on pourrait, à son tour, considérer responsable en dernière instance face aux législateurs. On pourrait répondre qu'ils échappent à la hiérarchie politique, mais que les normes qu'ils produisent restent dans le cadre de la hiérarchie normative qui leur a donné une habilitation qui les maintient, dans le cadre du système. Cette objection est tout à fait fondée si l'on considère la hiérarchie du point de vue statique, parce que la compatibilité, sinon la cohérence, reste assurée. mais, à mon avis, le caractère hiérarchique perd de son aspect dynamique: la décision de l'échelon inférieur ne peut plus être mise en rapport avec une source supérieure qui n'a pas un contenu concret à développer. En tout cas, la hiérarchie semble rester plutôt théorique en termes absolus, dans toute relation où deux sujets différents sont chargés l'un de la décision et l'autre de la mise en oeuvre. Comme le montre E. Blankenburg (58), la formulation de la décision a besoin d'anticiper les problèmes de la mise en oeuvre pour fixer des directives claires, et les chargés de la mise en oeuvre sont les mieux placés pour choisir les problèmes qu'ils veulent que l'on tienne en compte dans la décision "supérieure".

La clé serait, alors, le contrôle, et c'est aux juges que l'on pense tout de suite. Les juges sont tenus d'assurer le respect de l'ordre normatif, mais s'ils doivent assurer le principe de légalité, ils partent aussi de la présomption du respect de ce principe de la part des autorités ayant le pouvoir normatif ' II y a, alors, la possibilité que les juges portent cette présomption jusqu'au point de céder leurs fonctions d'interprétation et application des lois au profit des normes inférieures et, indirectement des autorités qui les produisent (59). La définition de la loi par le législateur et son application par le juge ne sont pas capables d'assurer l'efficacité des normes dans le processus de mise en oeuvre. La capacité régulatrice des pouvoirs publics est donc faible par rapport à l'administration, qui est l'appareil étatique en rapport immédiat avec la société. II est difficile de croire aux capacités régulatrices de l'État si ses propres agents ne sont pas contrôlables de façon effective. En tout cas, il est douteux que d'autres instances de contrôle social soient plus efficaces que celles des législateurs et des juges.

G. E. Frug (60), en faisant une référence a la doctrine américaine qui semble valable ailleurs, analyse de façon pessimiste les possibilités d'un contrôle effectif, et 
présente deux modèles de contrôle, à côté du modèle juridictionnel et du modèle "formel", des législateurs sur les administrateurs. Le modèle "expert" a une efficacité interne. II se fonde sur l'esprit de corps des administrateurs, qui se contrôleraient entre eux pour préserver les qualités et le prestige du métier en vieillant sur la performance. La viabilité de ce contrôle serait en principe facilitée par la prédisposition psychologique de ceux qui mettent leur sens de la responsabilité professionnelle au-dessus de tout autre attachement ou fidélité; on pourrait évoquer ici I'"effet Becket" décrit par B. François pour décrire la prééminence du rôle institutionnel (61) en ce qui concerne les juges. Mais le contrôle interne ne se fonde pas seulement sur le sens du devoir des administrateurs; il est possible du moment que la performance peut prendre comme référence des standards techniques propres au savoir du métier (62). Or, il y a des doutes sur le statut scientifique des sciences sociales, auxquelles appartient la science de l'administration; Frug cite Alasdair Macintyre, lequel qualifie le concept d'efficacité "managérielle" comme une fiction morale qui tient grâce aux capacités histrioniques des bureaucrates (63). L'autre modèle présenté par Frug est le modèle "pluraliste" (64): les administrateurs sont soumis au législateur, mais ils doivent aussi admettre les intéressés dans le processus de prise de décisions. II faut donc déterminer la condition d'intéressé, choisir qui va représenter chaque groupe d'intérêt et sous quels critères, et établir des normes de procédure pour régler les conflits dans le processus de prise de décisions. En plus, les intéressés potentiels n'arrivent pas toujours à être informés, surtout dans le cas des groupes marginalisés, ce qui fait des doutes sur l'efficacité d'une procédure de contrôle qui dépend de la diffusion de l'information et de la disponibilité à la mobilisation, outre les problèmes de désignation des parténaires.

\section{CONCLUSION}

On attribue a Ronald Reagan une phrase qui pourrait être la conclusion synthétique des secteurs libéraux les plus radicaux: "Le gouvernement n'est pas la solution; il est le problème". En d'autres mots, l'intervention publique est inefficace, sinon perverse. Dans les pages précédentes, j'ai essayé de décrire la crise de la régulation étatique. Une crise qui était annoncée au début du siècle par Durkheim et Santi Romano, comme j'ai déjà dit au commencement de ce travail, mais que le développement de l'État providence et sa constitutionnalisation sous forme d'État social semblait contredire. Aujourd'hui, la régulation étatique apparaît comme excessive; en tout cas, la tendance serait d'y échapper: "L'État, les groupes, les associations et les organisations bureaucratiques chercheraient de plus en plus à atteindre leurs objectifs de coordination et de contrôle par des modes de régulation plus 'neutres' (la technique ... ) et/ou plus axiologiques (la morale ... ) que les modes de régulation juridique" (65). Ces lignes représentent assez bien le désir d'af faiblissement du poids de la régulation étatique, tout en laissant soulevée la question de savoir si la régulation non étatique cesse d'être juridique. On rêve de 
l'autorégulation des groupes sociaux, tout en mettant à nouveau sur le tapis l'idée du "droit social" de Georges Gurvitch (66). Néanmoins, Gurvitch affirme que "la validité du droit social ne s'étend pas plus loin que les limites du groupe qu'il intègre" (67), ce qui maintient soulevées les questions sur le droit étatique. Un modèle "dérégulateur" extrême peut imaginer une sorte de fédération de groupements sociaux autorégulés, et on aurait l'image juridique de la fragmentation corporatiste de la société. Mais elle serait une image avec des vides inacceptables, car les problèmes de l'environnement ou de la protection des consommateurs ne trouvent pas de solution effective dans le jeu du marché (68).

Le droit étatique des États qu'on commence a appeler "postl"-sociaux (69) fait l'objet d'un débat important. Ce droit qu'on a tendance à juger encombrant n'est plus utile comme instrument de transformation de la société en profondeur. Si l'on n'accepte pas que la régulation sociale soit le résultat de l'évolution spontanée et que l'on considère la planification impossible, la voie intermédiaire est représentée par ceux qui croient que le droit peut être encore un instrument de "guidage" (70). Comme instrument de "guidage" alternatif aux régulations substantives, la régulation étatique devrait se borner aux normes de procédure, en laissant aux individus ou aux groupes la détermination des contenus (71). Après avoir montré un aperçu des difficultés de la régulation étatique, toute recherche de formes de régulation alternative mérite d'être suivie attentivement. Mais cette perspective continue à poser des problèmes. D'une part, la régulation procédurale peut faire avancer la régulation étatique dans des domaines où elle en était absente (72), sans éviter la régulation jurisprudentielle. D'autre part, on peut voir facilement qu'il y a des domaines où un droit purement procédural qui renoncerait à la régulation substantive ne serait pas capable de contrôler des conséquences peu désirables. La fixation de standards de qualité dans le droit de la consommation ou dans le droit de l'environnement peuvent arriver trop tard si on laisse la responsabilité de sa création aux acteurs sociaux, même si leurs rapports sont réglés de façon efficace. En ce qui concerne le droit du travail, je crois que Romagnoli a raison quand il dit que "la déréglementation dans ses différentes acceptions, implique moins en fait une diminution du nombre des règles existantes qu'une modification des rapports existants entre leurs sources" (73). A mon avis, moins de régulation étatique ne signifie pas toujours moins de régulation substantive. La crise de la régulation étatique est évidente, mais l'alternative globale n'arrive pas à se dessiner assez nettement. 


\section{NOTES}

(1) DURKHEIM, E.: Le suicide, Paris, P.U.F., 1960, pp. 436-437 (la première édition est de 1897). Santi Romano faisait la leçon inaugurale de l'année académique 1909-1910 de l'Université de Pise. Cette leçon est intégrée dans le recueil ROMANO, S.: Lo stato moderno e la sua crisi, Milan, Giuffrè, 1969, dont le titre est tiré de celui de la leçon, pp. 526. La citation se trouve à la p. 15 et l'autre référence à la p. 23.

(2) Un apport récent au débat est celui de GINER, S. et CAMPS, V.: L'interès comú, Barcelone, Fundació Caixa de Barcelona, 1990 (n. 5 de la collection "Cultura i entorn").

(3) Voir à ce sujet CROZIER, M.: État modeste, État moderne. Stratégie pour un autre changement, Paris, Fayard, 1987, chap. 5 et 6.

(4) CROZIER, M., HUNTINGTON, S.P. et WATANUKI, J.: The Crisis of Democracy, New York, New York University Press. Voir aussi BRITTAN, S.: "The Economic Contradictions of Democracy". British Journal of Political Science, n. 5, 1975, pp. 129-159.

(5) Sur cette notion de "surcharge", voir KING, A.: "Overload: Problems of Governing in the 1970s". Political Studies, vol. XIII, 1975, pp. 284-297.

(6) DAHRENDORF, R.: "Effectiveness and Legitimacy: on the Governability of Democracies". Political Quaterly, vol. LI, n. 4, 1980, pp. 393-410 (p. 405), a Propos du chapitre de S.P. Huntington dans M. Crozier, S.P. Huntington et J. Watanuki, op. cit. La crainte de voir dans toute restriction du marché un pas vers le totalitarisme est exprimée par HAYEK, F.A. dans The Road to Serfdom, surtout dans les chaps. V, VI et VII (j'ai utilisé la version espagnole Camino de servidumbre, Madrid, Ed. Revista de Derecho Privado, 1946; l'édition originale est de 1943), et il insiste dans Droit, législation, liberté, Paris, Presses Universitaires de France, 1980-83, 3 vols., vol. II, p. 100. II est intéressant de mettre à côté de The Road to Serfdom (1943) son contemporain POLANYI, K.: The Great Transformation, New York, Rinehart and Co., 1941, chaps. XIX et XXI (j'ai utilisé la version française La grande transformation, Paris, Gallimard, 1983).

(7) voir la position sceptique de GIANNINI, M.S.: "Stato sociale, una nozione inutile" dans les Scritti in onore di Costantino Mortati, Milan, Giuffrè, 1977, 3 vols., vol I, pp. 144-165; il insiste dans II pubblico potere. Stati e amministrazioni pubbliche, Bologne, II Mulino, 1986, pp. 95-98.

(8) FLORA, P. et ALBER, J.: "Modernization, Democratization and the Development of Welfare State in Western Europe" dans P. Flora et A.J . Heidenheimer (eds.), The Development of Welfare States in Europe and America, New Brunswick (N.J.), Transaction Books, 1981, pp. 37-80 (surtout p. 70). Ainsi que les nuances de THERBORN, G.: "Class and States. Welfare State Developments, 1881-1981". Studies in Political Economy, n. 14, 1984, pp. 7-41 (surtout p. 12).

(9) DION, L.: "L'État libéral et l'expansion de l'espace public étatique". International Political Science Review, vol. VII, n. 2, 1986, pp. 190-208.

(10) Voir dans EWALD, F.: L'État providence, Paris, Grasset, 1986, les chapitres sur l'assurance. II faut dire que la protection sociale est vue comme un droit des citoyens, et non pas comme une charité qui leur est accordée, d'après WILENSKY, H.: The Welfare State and Equality. Structural and Ideological Roots of Public Expenditures, Berkeley, University of California Press, 1975, p. 1.

(11) Ce qui ne veut pas dire non plus que la notion de "droits sociaux" soit une notion récente. On peut la trouver dans l'intervention de Gilbert ROMME dans la séance de la Convention du 17 avril 1793, Archives parlementaires, Série I, vol. LXII, pp. 263-269. Et comme droits de prestation, ils peuvent s'intégrer dans la catégorie des droits publics subjectifs d'après JELLINEK, G.: Sistema dei diritti pubblici subbietivi, Milan, Società Editrice Libraria, 1912 (traduction de la 2ème. édition allemande de 1905; 1ère. édition 1892), pp. 97-98.

(12) Je prends l'expression comme étant équivalente à "welfare state". 
(13) Elle apparaît comme l'une des quatre libertés auxquelles le fameux discours de F.D. ROOSEVELT faisait allusion dans son message au Congrès des Etats-Unis du 6 janvier 1941: "F.D. Roosevelt's 'Four Freedoms' Speech", dans H. Commager (ed.), Documents of American History, New York, Appleton-Century, 1971, pp. 446-449. C'est aussi une idée clé dans le rapport Beveridge; voir BEVERIDGE, W.H.: Full Employment in a Free Society, Londres, George Allen \& Unwin, 1954 (1ère. édition est de 1944).

(14) La première formulation de la notion d'État social dans le sens qui nous est plus familier est de HELLER, H. dans "Rechtstaat oder Diktatur?"; j'ai employé la version espagnole publiée dans Escritos políticos, Madrid, Alianza Editorial, 1985, pp. 283-301 (pp. 288-289). Sur la relation entre Etat providence et Etat social ("welfare state" et "social state"), voir DALBERG-LARSEN, J.: The Welfare State and its Law, Berlin, Verlag Dr. Tesdorpf, 1987, pp. 8-16.

(15) MIRKINE-G-UETZEVICH, B.: Les Constitutions européennes, Paris, Presses Universitaires de France, 1951, 2 vols., vol. I, pp. 133 et ss.

(16) Voir la préface de GASS, J.R., directeur des affaires sociales, dans le rapport de l'OCDE: The Welfare State in Crisis. An Account on the Conference on Social Policies. OECD, Paris, 20-23 october 1980, Paris, OCDE, 1981, pp. 5-6. II y a, bien sûr, des vues différentes, qui établissent un lien entre protection sociale et efficacité économique (par exemple SEGURA, J.: "La reforma del Estado asistencial". Cuadernos y debates, n. 2, 1987, pp. 15-40, surtout pp. 19-20), ou qui présentent les politiques de protection sociale comme étant liées à la "raison d'État" contemporaine (WOLIN, S.: "The Political and Theoretical Connections between Staatsräson and Wohlfahrsaatsräson". Political Theory, vol. XV, n. 4, 1987, pp. 467-500, surtout pp. 480 et 495-496).

(17) DE JASAY, A.: The State, Oxford, Basil Blackwell, 1985, p. vii.

(18) WEBER, M.: Economia y sociedad, México, Fondo de Cultura Económica, 1979, p. 653 (Part II, Chap. VIII). Voir aussi, NEUMANN, F.: "The change in the function of law in modern society" dans son livre The Democratic and the Autoritarian State. Essays in Political and Legal Theory, New York, Free Press, 1957.

(19) Voir FORSTHOFF, E.: "La transformazione della legge costituzionale" dans son livre Stato diritto in trasformazione, Milan, Giuffrè, 1973, pp. 195-232. La version italienne est faite d'après l'allemande de 1964. Voir aussi DE OTTO, I.: Derecho constitucional. Sistema de fuentes, Barcelone, Airel, 1987, pp. 81-82.

(20) Surtout dans le domaine du social; voir KAHN-FREUND, O.: Trabajo y Derecho, Madrid, Ministerio de Trabajo y Seguridad Social, 1987 (traduction de l'édition anglaise de 1983), p. 48.

(21) DOGLIANI, M.: Interpretazioni della Costituzione, Milan, F. Angeli, 1982 (surtout pp. 7-28). Voir aussi GOMES CANOTILHO, J.J.: Constituiçao dirigente e vinculaçao do legislador, Coimbra, Coimbra Editora Limitada, 1982, et NIETO, A.: "El positivismo jurídico y la Constitución de 1978". Revista Española de Derecho Constitucional, vol. IX, n. 26, 1989, pp. 9-39 (pp. 9-16).

(22) Les problèmes de la "juridification" (Verrechtlichung) sont traités par HABERMAS, J.: Theorie des kommunicatives Handels. J'ai utilisé la version espagnole Teorla de la acción comunicativa, Madrid, Taurus, 1987, 2 vois., vol. II, pp. 502-527.

(23) CARBONNIER, J.: Flexible droit, Paris, Libraire Générale du Droit et de la Jurisprudence, 1969, pp. 20-49. Le "non-droit" est "l'absence du droit dans un certain nombre de rapports humains où le droit aurait eu une vocation théorique à être présent" (p. 20).

(24) Voir KOOPMANS, T.: "Legislative and judiciary. The present trends", dans M. Cappelletti (ed.), New Perspectives for a Common Law of Europe, Florence, Le Monnier, 1975, pp. 309-337. Aussi, FERRAJOLI, L.: "La crisi dello Stato di diritto nella crisi dello Stato sociale", dans E. Fano, S. Rodotà et G. Marramao (eds.), Transformazioni e crisi del Welfare State, Bari, De Donato/Regione Piemonte, 1983, pp. 419-429. Ferrajoli nie la spécificité de l'État social par rapport à l'État de droit. Une critique des analyses de la "surcharge" qui partent de l'autonomie du système juridique, FRIEDMAN, L.M.: "The Limits of Law: a Critique and a Proposal", Diskussionbeiträge n. 80, 1986, Université de Siegen, Centre d'études sur la mobilité historique et le changement des normes. 
(25) Voir DE OTTO, I.: "La función politica de la legislación", dans M.A. APARICIO (coord.), Parlamento y sociedad civil, Barcelona, Publicaciones de la Universidad de Barcelona, 1980, pp. 49-59. Aussi HABERMAS, J.: L'espace public, Paris, Payot, 1978, pp. 89-98 (l'édition originale allemande est de 1962).

(26) VON BEYME, K.: "The Role of the State and the Growth of the Government". International Political Science Review, vol. VI, n. 1, 1985, pp. 11-34.

(27) NIETO, A.: La organización del desgobierno, Barcelona, Àriel, 1984, surtout pages 5178.

(28) MIRKINE-GUETZEVICH, B.: op. cit., vol. I, pp. 18-29 et GALLEGO ANABITARTE, A.: Ley y reqlamento en el Derecho Público occidental, Madrid, Instituto de Estudios de Administración Local, 1971 , pp. $217-219$ et $335-340$.

(29) Du moment où selon la Section 1 de l'article I de la Constitution des Etats-Unis il est établi que tous les pouvoirs législatifs sont octroyés ("vested") au Congrès. On cite souvent à ce propos le chapitre XI de l'Essai sur le gouvernement civil de LOCKE, John, et la maxime delegata potestas delegari non potest.

(30) J'ai traité ce problème dans ARBOS, X.: "Un hito en la jurisprudencia constitucional norteamericana: la sentencia 'Inmigration and Naturalisation Service vs. Chadah' y el 'legislative veto'". Revista de Estudios Políticos, n. 34, 1984, pp. 229-236. Une approche générale sur les problèmes de la dérégulation dans SCHMIDT, V.A.: "La dérégulation aux Etats-Unis". Revue française d'Administration publique, n. 41, 1981, pp. 115-127.

(31) ROSE, R.: "Inheritance before Choice in Public Policy". Journal of Theoretical Politics, vol. II, n. 3 , 1990, pp. 263-291 (p. 268).

(32) CARRÉ DE MARLBERG, R.: La loi, expression de la volonté générale, Paris, Economica, 1984 (reproduction du texte publié par Sirey en 1931).

(33) Sur les problèmes liés à la distribution du pouvoir normatif, voir BARCELLONA, P.: I soggetti e le norme, Milan, Giuffrè, 1984, n. 16 de la "Biblioteca per la storia del pensiero giuridico moderno", surtout pp. 142-143.

(34) Sur la crise de la codification, voir IRTI, N.: L'età della decodificazione, Milan, Giuffrè, 1979 et CLAVERO, B.: "Codificación y constitución: paradigmas de un binomio". Quaderni fiorentini per la storia del pensiero giuridico moderno, n. 18, 1989, pp. 79-145 (pp. 124-134). Sur la diversification formelle de la loi et la complexité étatique, GARCIA PELAYO, M.: Las transformaciones del Estado contemporáneo. Mádrid, Alianza Editorial, 1977, pp. 162-163. Sur les lois singulières, ARIÑO ORTIZ, G.: "Leyes singulares, leyes de caso único". Revista de Administración Pública, n. 118, 1989, pp. 57-101. J'emploie les notions de "loi spéciale" et "loi singulière" de façon différente de DE OTTO, I.: Derecho constitucional. Sistema de fuentes, op. cit., pp. 179-180. La Cour constitutionnelle espagnole a admis la constitutionnalité des "lois singulières" sous certaines conditions: que la "loi singulière" réponde à la singularité des circonstances de fait et qu'elle soit un moyen raisonnable d'obtenir le résultat voulu par le législateur et admissible par la Constitution (STC 166/1986, du 19 décembre, FJ 10 et 11).

(35) IRTI, N.: op. cit., pp. 27-28.

(36) GINER, S.: "Clase, poder y privilegio en la sociedad corporativa", chap. 3 de son livre Ensayos civiles, Barcelone, Península, 1987, pp. 79-135 (surtout pp. 103-112).

(31) ARIFJO ORTIZ, G.: op. cit., p. 57.

(38) HAYEK, F.A.: The Constitution of Liberty, Chicago, Chicago University Press, 1960, pp. 153-154.

(39) C'est le cas de la Constitution italienne (art. 3) et de la Constitution espagnole (art. 9.2.). 
(40) DIAZ, E.: Estado de derecho y sociedad democrática, Madrid, Taurus, 1981 (lére. ed. 1966).

(41) BENTHAM, J.: Tactique des Assemblées politiques délibérantes, chap. III "De la publicité", pp. 382-389 du vol. I de ses Oeuvres, réimpression de l'édition de Bruxelles 1829, trad. de P.E.L. Dumont et B. Laroche, Darmstadt, Scientia Verlag Aalen, 1969.

(42) RESCIGNO, G.U.: "Appendice" (fragments du rapport au Parlement italien sur l'amélioration des lois), dans PAGANO, R. (coord.): Normative europee sulla tecnica legislativa, 2 vol., Rome, Camera dei Deputati, 1988, (coli. "Quaderni di documentazione" n. 10), vol. I, pp. 349-365 (p. 357).

(43) NITSCH, N.: "L'inflation juridique et ses conséquences". Archives de Philosophie du droit, vol. XXVII, 1982, pp. 161-179 (pp. 167169).

(44) Une vision futuriste dans SCHMITTER, P.C.: "Neocorporatismo y Estado". Revista española de investigaciones sociológicas, n. il, 1985, pp. 47-48 (pp. 72-73).

(45) BURDEAU, G.: "Une survivance: la notion de Constitution", dans l'ouvrage collectif L'évolution du droit public. Etudes en honneur d'Achille Mestre, Paris, Sirey, 1956, pp. 53-62 (pp. 57-58). Cfr. BURDEAU, G.: "Constitution, droits de l'homme et 'changement'", dans l'ouvrage collectif $\underline{\text { Scritti in }}$ onore di Vzio Crisafulli, 2 vol., Padoue, CED , 1985, vol. II, pp. 119-126.

(46) LOPEZ DE ODATE, F.: La certezza del diritto, Milan, Giuffrè, 1968, surtout pp. 4578.

(47) IRTI, N.: op. cit., pp. 95-97; la citation est de la p. 97. A ce sujet, l'Association Internationale de Droit constitutionnel a tenu un colloque a Varsovie sous le titre "Le rôle du droit constitutionnel dans la prévention de l'inflation normative" du 16 au 18 octobre 1985. Voir aussi DEBBASCH, C. (coord.): L'inflation législative et réglementaire en Europe, Paris, C.N.R.S., 1986.

(48) Sur la question dans la jurisprudence constitutionnelle allemande, BOCANEGRA SERRA, R.: El valor de las sentencias del Tribunal constituclonal, M-adrîd, I.E.A.L., 1982, pp. 240-248. Voir en général PORRAS NADALES, A.J.: "El derecho intervencionista del Estado". Revista de Estudios Políticos, n. 63, 1989, pp. 65-82 (p. 81). En Espagne, la doctrine de la Cour Constitutionnelle est exposée clairement dans la STC 45/1989, du 20 février, FJ 11.

(49) SUBIRATS, J.: Análisis de políticas públicas y eficacia de la Administración, Madrid, Instituto Nacional de Administración Pública, 1989, p. 26, rappelle cue "la 'jungla normativa' permite todo tipo de adecuación 'al caso' de la normativa, aplicando la más conveniente, ignorando la que no interesa o supliendo una determinada laguna por una interpretación ad hoc". On pourrait nuancer cette image par les contrôles de constitutionnalité et de légalité, qui assurent la "non contradiction" verticale de la hiérarchie de l'ordonnancement juridique. Et, en tout cas, il serait aussi possible de dire que si la jungle normative permet de tout faire aux administrateurs, elle permet aussi à la juridiction de tout annuler, car on peut toujours trouver une règle qui aurait été violée. Voir CROZIER, M. et FRIEDBERG, E.: L'acteur et le système, Paris, Ed. du Seuil, 1977, pp. 88-90.

(50) Sur l'ensemble légitimité/efficacité, voir LIPSET, S.M.: Political Man, New York, Doubleday \& Co., 1960 , pp. 77-83. Les hommes politiques le savent très bien: ROCARD, M. présentait la "performance" et la "légitimité" comme étant les deux impératifs de l'action politique, dans Le coeur à l'ouvrage, Paris, Odile Jacob, 1987, p. 151.

(51) KNAPP, V.: La scienza del diritto, Rome-Bari, Laterza, 1978 (traduction italienne du rapport de I'Unesco du même titre de 1976), surtout pp. 50-72. La préface de GIANNINI, M.S. est aussi très intéressante, surtout pp. XXXI-XLVII. Voir aussi CALSAMIGLIA, A.: "Justicia, eficiencia y optimización de la legislación". Documentación Administrativa, n. 218-219, 1989, pp. 113-151.

(52) KIRCHHEIMER, O.: "The 'Rechstaat' as a Magic Wall", dans K.H. Wolff et B. Moore jr. (eds.), The Critical Spirit. Essays in Honor of Herbert Marcuse, Boston, Beacon Press, 1967, pp. 287-312.

(53) Sur le droit comme instrument d'ingénierie sociale, voir ZULETA PUCEIRO, E.: "La función del Derecho en el Estado social". Revista general de legislación y jurisprudencia, n. 6, 1983 pp. 555-568 (p. 567), et OFFE, C.: "Reflections on the welfare state and the future of socialism" (interview avec 
D. Held et J. Keane en 1982) dans son ouvrage Contradictions of the welfare state, Londres, Hutchinson, 1984, pp. 252-299 (pp. 280-281).

(54) Sur ces questions, voir GARCIA PELAYO, M.: Burocracia y tecnocracia, Madrid, Alianza Editorial, 1982 (1ère. éd. 1974), pp. 28-32. Aussi BERTI, G.: Diritto e Stato. Riflessioni sul cambiamento, Padoue, CEDAM, 1986, p. 90.

(55) Sur les modèles wéberiens, voir COHEN, P.S.: "Rational conduct and social life", dans S.I. Benn et G.W. Mortimore (eds.), Rationality and the social sciences, Londres, Henley et Boston, Routledge and Kegan Paul, 1976, pp. 132-154 (pp. 133-135). Ces affirmations s'encadrent dans l'État de droit, où le principe de légalité oblige à des raisonnements fondés seulement sur des normes, et dans ce sens ils sont des raisonnements "clos" selon FRIEDMAN, L.W.: Law and society. An introduction, Englewood Cliffs, Prentice Hall, 1977, pp. 80-83.

(56) SANTAMARIA PASTOR, J.A.: Fundamentos de Derecho administrativo, vol. I, Madrid, Ed. Centro de Estudios Ramón Areces, 1988, pp. 727-732. La jurisprudence constitutionnelle espagnole est réduite dans ce domaine, et elle n'a pas encore abordé dans le problème des effets extérieurs: STC 26/1986, du 19 février, FJ 1.

(57) En Espagne la question est encadrée par la loi 24/1988 du 28 juillet, sur la régulation du marché des valeurs mobilières, art. 15 (Réf. Aranzadi 1644) et la loi 26/1988, du 29 juillet, sur la régulation des entités de crédit, art. 1.5. Les articles suivants sont encore utiles, parce que les problèmes qui y sont traités se posent encore: PARADA VAZQUEZ, J.R.: "Valor jurídico de la circular". Revista de Derecho bancario y bursátil, vol. I, n. 2, 1981, pp. 311-320, et FERNANDEZ RODRIGUEZ, T.R.: "Los poderes normativos del Banco de Españla", ibid., vol. IV, n. 13, 1984, pp. 732.

(58) BLANKENBURG, E.: "The Waning of Legality in the Concept of Policy Implementation". Law \& Policy, vol. VII, n. 4, 1985, pp. 481-491, surtout p. 490. Voir aussi SUBIRATS, J.: "El papel de la burocracia en el proceso de determinación e implementación de las políticas públicas". Revista vasca de Administración pública, n. 25, 1989, pp. 67--76. Comparer avec MARINO, I.: "Aspetti giuridici della programmazione: programmazione e mete sociali". Diritto e Società, n. I., 1990, pp. 21-53 (pp. 28-31).

(59) PRIEUR, J.: "Jurisprudence et séparation des pouvoirs". Archives de philosophie du droit, vol. XXX, 1985, pp. 117-130. En Espagne, MUNOZ MACHADO, S.: La reserva de jurisdicción, Madrid, La Ley, 1989, pp. 109-111. Sur le problème des circulaires, PRIEUR, J.: art. cité, p. 121.

(60) FRUG, G.E.: "The Ideology of Bureaucracy in American Law". Harvard Law Review, vol. XCVII, n. 6, 1984, pp. 1276-1388.

(61) FRANÇOIS, B.: "Le juge, le droit et la politique". Revue française de Droit constitutionnel, n. 1, 1990, pp. 49-69 (p. 54), rappelle que Thomas Becket a servi les intérêts de l'Eglise contre ceux du roi Henri II Plantagenêt.

(62) WEBER, M.: op. cit., pp. 179-180. où il fait référence à la rationalité matérielle. La définition et l'application du droit contemporain intègre souvent des valeurs et des objectifs sociaux, ce qui constitue l'une de ses caractéristiques selon ERIKSSON, L.D.: "Conflicting Tendencies in Modern Law". Rechtstheorie, n. 2, 1989, pp. 153-162 (p. 154).

(63) MACINTYRE, A.: After virtue, Notre Dame, University of Notre Dame Press, 1984, chap. 8. j'ai utilisé la version espagnole, Tras la virtud, Barcelone, Crítica, 1987 (pp. 138 et 139).

(64) En fait, FRUG présente et analyse sur le même plan le modèle du marché par rapport au contrôle sur les "managers" et le modèle pluraliste (pp. 1355-1377). II faut souligner qu'il ne les présente pas comme des contrôles opérationnels; voir COHEN-TANUGI, L.: Le Droit sans l'État. Sur la démocratie en France et en Amérique, Paris, P.U.F., 1987, pp. 116-121.

(65) BELLEY, J.G.: "L'État et la régulation juridique des sociétés globales. Pour une problématique du pluralisme juridique". Sociologie et sociétés, vol. XVIII, n. 1, 1986, pp. 11-32 (pp. 30-31). 
(66) ROSANVALLON, P.: La crise de l'État providence, Paris, du Seuil, 1981, p. 116.

(67) Dans l'ouvrage auquel fait référence P. ROSSANVALLON, GURVITCH, G.: L'idée du droit social, Paris, Sirey, 1932 (j'ai utilisé la reproduction publiée a Darmstadt, Scientia Verlag Aalen, 1972), et la notion de droit social est présentée pp. 15-35 (la citation, p. 21). Sur ce point des réflexions de Gurvitch, voir FERNANDEZ, E.: "Derecho social, democracia y pluralismo en G. Gurvitch". Sociología y psicología jurídicas, 1987, pp. 5-17.

(68) MARTIN MATEO, R.: Liberalización de la Economía. Más Estado, menos Administración, Madrid, Trivium, 1988, pp. 86-87.

(69) PORRAS NADALES, A.J.: Introducción a una teoria del Estado postsocial, Barcelona, PPU, 1988. Une perspective générale sur le débat contemporain à ce sujet, TEUBNER, $G$. (ed.): Dilemmas of law in the contemporary state, Berlin, de Gruyter, 1985.

(70) WILKE, H.: "Diriger la societé par le droit?". Archives de philosophie du droit, vol. XXXI, 1986, pp. 189-214. II faut dire que Wilke exclut la possibilité de contrôler directement les sous-systèmes des sociétés complexes par des politiques administratives ou juridiques (p. 205); on peut entendre que parmi ces sous-systèmes se trouve l'économie et le droit lui-même (p. 191).

(71) PREUSS, U.K.: "La crisis del mercado de trabajo y las consecuencias para el Estado social", dans CORCUERA ATIENZA, J. et GARCIA HERRERA, M.A. (coords.): Derecho y economía en el Estado social, Madrid, Tecnos, 1988, pp. 71-94 (P. 91). voir aussi TEUBNER, G.: "Substantive and Reflexive Elements in Modern Law". Law and Society Review, vol. XVII, n. 2, 1983, pp.239-285.

(72) BLANKENBURG, E.: "The Poverty of Evolutionism: A Critique of Teubner's case for 'Reflexive Law'". Law and Society Review, vol. XVIII, n. 2, 1984, pp. 273-289 (p. 285).

(73) ROMAGNOLI, U.: "La déréglementation et les sources du droit du travail". Revue internationale de Droit comparé, n. 1, 1990, pp. 9-25 (p. 18). 